\title{
Patient's Perception in Bicruciate-Retaining Total Knee Arthroplasty
}

\author{
Florian Baumann*1 and Himanshu Bhayana ${ }^{2}$ \\ ${ }^{1}$ Department of Trauma Surgery, Regensburg University Medical Center, Germany \\ ${ }^{2}$ Department of Orthopedics', UCMS and GTB Hospital, India
}

Received: March 19, 2018; Published: March 28, 2018

${ }^{*}$ Corresponding author: Florian Baumann, Department of Trauma Surgery, Regensburg University Medical Center, Germany, Tel: 0941/944-6805; Email: Florian.Baumann@klinik.uni-regensburg.de

Keywords: Bicruciate-Retaining Total Knee Arthroplasty (BCR-TKA); Outcome; Proprioception; Knee Kinematics

Abbreviations: TKA: Total Knee Arthroplasty; ACL: Anterior Cruciate Ligament; : ADL: Activity Of Daily Living; ROM: Range of Motion; PROM: Patient-Reported Outcome Measurements; FJS: Forgotten Joint Score

\section{Introduction}

In treatment of advanced osteoarthritis, total knee arthroplasty (TKA) with the sacrifice of the anterior cruciate ligament (ACL) is the standard treatment. There is high proportion of patients who report residual knee symptoms after TKA without any identifiable objective clinical or radiological reasons. In regards to cruciate-retaining knee arthroplasty in the last decade, knee surgeons have mainly focused on the posterior cruciate ligament [1-4]. However, anatomic studies have shown cruciate ligaments; especially anterior cruciate ligament contains a considerable number of proprioceptive nerve cells. Sacrifice of the ACL can also be accompanied with abnormal kinematics resulting in functional limitations and a reduced balance [3,4]. Reduced balance capacity can cause various problems in activity of daily living (ADL). Balance problems due to limitations in proprioception after TKA can cause frequent falls. Besides this, reduced balance after the arthroplasty can lead to a situation where patient senses the joint as a foreign body which can be associated with non-specific pain and can cause functional deficit [1,4-6]. First generation bicruciateretaining total knee arthroplasty (BCR-TKA) was developed in the 1960s which aimed for a natural knee joint movement. Bicruciateretaining implants have shown a more physiologic anterior femorotibial contact point and a greater posterior translation in motion compared to ACL-sacrificing implants Cloutier et al.[1,3,6-10].

Reported excellent clinical results for the first generation of BCR-TKA with a survivorship rate of $95 \%$ after ten years and $82 \%$ after 22 years. However $38 \%$ of patients had limited range of motion (ROM) and pain. Despite technical advances, many patients have reduced ROM compared to standard TKA [11]. This problem is especially common in patients who have limited preoperative ROM [12]. Initial results of BCR-TKA have shown that these implants are associated with a higher "overall satisfaction" [13]. However, the question remains if this improvement is due to retention of ACL. Proprioception is the ability to sense the relative position and movement of neighboring anatomic structures. Schwartz et al have found balance ability an important factor for quality of life after TKA [14]. Therefore, the question is whether the bicruciate-retaining implant can provide superior balance ability and improved quality of life. In a recent prospective cohort study, we found that bicruciateretaining implants can provide a superior level of proprioception compared to standard cruciate-sacrificing implants [11].

Besides proprioceptive abilities, the patient's sensation of the artificial joint is crucial for the patient's perception. Prior studies on patient satisfaction after TKA have used conventional clinical scores (e.g. Knee Society Score [15]. Conventional scores have impairments in discrimination of good and very good results. This makes it difficult to reveal minor differences in TKA. However, recent studies have shown that BCR-TKA is perceived as "overall better" compared to a cruciate sacrificing TKA $[6,13,16]$. Patient-reported outcome measurements (PROM) have become an important tool to evaluate activities, limitations in everyday life and quality of life. Several scores have proven their validity to discriminate patients with a good or excellent outcome from patients with a poor outcome very well. The Forgotten Joint Score (FJS) is a relatively new PROM tool paying special attention on the patient's ability to forget the 
artificial joint in everyday life. Accordingly, the loss of awareness of the artificial knee joint is seen as the ultimate goal resulting in maximum patient satisfaction. Recently published data on PROM of patients after BCR-TKA confirmed a reduced joint awareness for BCR-TKA compared to a standard total knee arthroplasty [16].

The FJS score values of the BCR-group were equal to the unicondylar knee arthroplasty patients. These results support the assumption that a bicruciate-retaining knee arthroplasty provides a superior patient perception. Nevertheless, implantation of a BCR-TKA is a technically demanding procedure. There are reports of a long learning curve even for experienced knee surgeons [17]. There are also reports on intraoperative island fractures due to a narrow bony base of the ACL and postoperative cyclops syndromes $[12,18,19]$. Development of these implants is associated with additional costs. The question is whether companies are willing to take the risks of development costs given that the product currently has a limited target group of patients.

\section{Conclusion}

BCR-TKA is technical advancement for patients with knee OA showing early promising results regarding postoperative function and patient satisfaction. However, these results are related to some basic requirements like patient selection and the surgeon's experience with the implant. Further prospective randomized trials are necessary to investigate long-term survivorship and limitations in early postoperative range of motion of some patients.

\section{References}

1. Heyse TJ, Slane J, Peersman G, Dirckx M, van de Vyver A, et al. (2017) Kinematics of a bicruciate-retaining total knee arthroplasty. Knee surgery, sports traumatology, arthroscopy 25(6): 1784-1791.

2. Hossain FS, Konan S, Patel S, Rodriguez Merchan EC, Haddad FS (2015) The assessment of outcome after total knee arthroplasty: are we there yet? The bone \& joint journal 97-B(1): 3-9.

3. Koh YG, Son J, Kwon SK, Kim HJ, Kwon OR, et al. (2017) Preservation of kinematics with posterior cruciate-, bicruciate- and patient-specific bicruciate-retaining prostheses in total knee arthroplasty by using computational simulation with normal knee model. Bone \& joint research 6(9): 557-565.

4. Lewis GN, Rice DA, McNair PJ, Kluger M (2014) Predictors of persistent pain after total knee arthroplasty: a systematic review and metaanalysis. British journal of anaesthesia 114(4): 551-561.

5. Andrawis J, Akhavan S, Chan V, Lehil M, Pong D, et al. (2015) Clinical orthopedics' and related research 473(8): 2688-2697.
6. Ward TR, Burns AW, Gillespie MJ, Scarvell JM, Smith PN (2011) Bicruciate-stabilised total knee replacements produce more normal sagittal plane kinematics than posterior-stabilised designs. The Journal of bone and joint surgery British volume 93 (7): 907-913.

7. Komistek RD, Allain J, Anderson DT, Dennis DA, Goutallier D (2002) In vivo kinematics for subjects with and without an anterior cruciate ligament. Clinical orthopedics' and related research 404: 315-325.

8. Peersman G, Slane J, Dirckx M, Vandevyver A, Dworschak P, et al. (2017) The influence of polyethylene bearing thickness on the tibiofemoral kinematics of a bicruciate retaining total knee arthroplasty 24(4): 751760 .

9. Cloutier JM (1983) Results of total knee arthroplasty with a nonconstrained prosthesis. The Journal of bone and joint surgery American volume 65 (7): 906-919.

10. Sabouret P, Lavoie F, Cloutier JM (2013) Total knee replacement with retention of both cruciate ligaments: a 22-year follow-up study. The bone \& joint journal 95-B(7): 917-922.

11. Baumann F, Bahadin O, Krutsch W, Zellner J, Nerlich M, et al. (2017) Proprioception after bicruciate-retaining total knee arthroplasty is comparable to unicompartmental knee arthroplasty. Knee surgery sports traumatology arthroscopy 25(6): 1697-1704.

12. Lavoie F, Al-Shakfa F, Moore JR, Mychaltchouk L, Iguer K (2017) Postoperative Stiffening after Bicruciate-Retaining Total Knee Arthroplasty. The journal of knee surgery.

13. Pritchett JW (2011) Patients prefer a bicruciate-retaining or the medial pivot total knee prosthesis. The Journal of arthroplasty 26 (2): 224-228.

14. Schwartz I, Kandel L, Sajina A, Litinezki D, Herman A, et al. (2012) Balance is an important predictive factor for quality of life and function after primary total knee replacement. The Journal of bone and joint surgery British volume 94 (6): 782-786.

15. Insall JN, Dorr LD, Scott RD, Scott WN (1989) Rationale of the Knee Society clinical rating system. Clinical orthopaedics and related research (248): 13-14.

16. Baumann F, Krutsch W, Worlicek M, Kerschbaum M, Zellner J, et al. (2018) Reduced joint-awareness in bicruciate-retaining total knee arthroplasty compared to cruciate-sacrificing total knee arthroplasty. Archives of orthopaedic and trauma surgery 138 (2): 273-279.

17. Christensen JC, Brothers J, Stoddard GJ, Anderson MB, Pelt CE, et al. (2017) Higher Frequency of Reoperation with a New Bicruciateretaining Total Knee Arthroplasty. Clinical orthopaedics and related research 475(1): 62-69.

18. Klaassen MA, Aikins JL (2017) The cyclops lesion after bicruciateretaining total knee replacement. Arthroplasty today 3(4): 242-246.

19. Behrend H, Giesinger K, Giesinger JM, Kuster MS (2012) The forgotten joint as the ultimate goal in joint arthroplasty: validation of a new patient-reported outcome measure. The Journal of arthroplasty 27(3): 430-436.

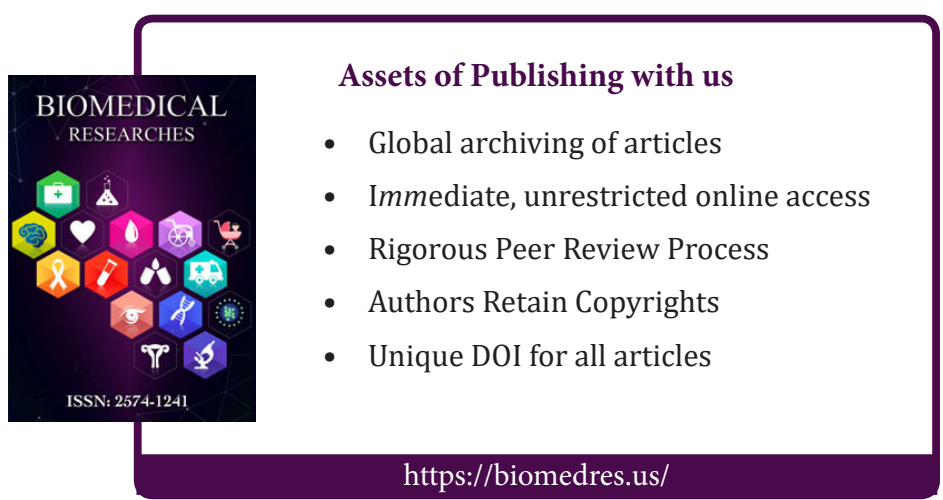

